\title{
CALIBRATION OF MACHINE VISION SYSTEM FOR NONDESTRUCTIVE DETECTION OF PLANTS
}

\author{
Ming Sun ${ }^{1,2}$, Dong An ${ }^{1}$, Yaoguang $\mathrm{Wei}^{1}$ \\ ${ }^{1}$ College of Information and Electrical Engineering, China Agricultural University, Beijing, \\ China, 100083 \\ ${ }^{2}$ Author for correspondence Address, P.O. Box 63, 17 Tsinghua East Road, Haidian \\ District, Beijing, 100083, P. R. China, Tel: +86-10-62737591, Email: drmingsun@163.com
}

\begin{abstract}
The detection of plants based on a machine vision system is one of the most important parts of plants simulation. The calibration of the machine vision system is a basic step for nondestructive detection. We discuss common methods such as traditional calibration techniques, active vision based calibration techniques, and self-calibration techniques. The results show that it is feasible to apply the traditional linear calibration method in nondestructive measurement of plants.
\end{abstract}

Keywords: machine vision, calibration, nondestructive detection

\section{INTRODUCTION}

Machine vision is a focus of technologies, which is used to simulate the outside or macroscopically vision function of the target by the study of computer's applications. Its chief function is to create or renew the real object by images. Machine vision technique is widely applied to industry, agriculture, spaceflight, remote sensing and medicine, etc. because of its contactless detection, fast speed and high precision. Recently, it also shows great application prospects in botany, agronomy, ecology and forestry (Jain et al., 2003).

Sun, M., An, D. and Wei, Y., 2008, in IFIP International Federation for Information Processing, Volume 259; Computer and Computing Technologies in Agriculture, Vol. 2; Daoliang Li; (Boston: Springer), pp. 1311-1314. 
The purpose of the detection of plants based on machine vision system is to study the growing rule of plants quantitatively. The calibration of the machine vision system is a basic step for nondestructive detection, which means that the calibration of the camera in the machine vision system is also necessary. The calibrating techniques of camera can be divided into traditional calibration, self-calibration, and active vision based on calibration. We choose one, which is adapting to the nondestructive detection of plants.

\section{METHODS FOR CAMERA CALIBRATION}

The methods for camera calibration can be divided into traditional calibration techniques, self-calibration techniques, and active vision based on calibration techniques. The above-mentioned methods will be discussed respectively.

Traditional calibration techniques is to use the frame information of the scenery which usually takes a calibration block whose frame is known and process precision is high as a directional reference, and to simulate the relation between the parameters of the camera model through the corresponding relation of a space spot and a camera spot, and then to get the parameter by optimizing algorithm. The calibration method can use any kind of camera model and has a high precision. But it requests a high-precision calibration block, and we cannot use the block in practical applications sometimes. This method mainly includes direct linear transform camera calibration (Yang et al., 2000), calibration based on the RAC (Tsai, 1987), and planar calibration (Zhang, 1998; Wu and Sun, 2004).

The camera calibration based on active vision is an important branch of the camera calibrations. The so-called camera calibration based on active vision is a method to calibrate cameras in a condition that some movement information of the camera is known, and the movement information includes the quantitative information and the qualitative information. The quantitative information refers to the case that the camera was translated for a given distance to a certain direction in the reference frame of the platform, and the two-translation movement orthogonality of the camera, etc. The qualitative information refers to the case that the camera does absolute linear movement or absolute rotary movement, etc. The main advantage of the camera calibration based on active vision is introduced as follows: The model parameters of the camera can be evaluated by linear equations since the movement information of the camera in the calibration process is known. The current research focuses on evaluating the model parameters through linear equations while reducing the movement restriction of the camera as much as possible. Representative researches include calibration based on three-orthogonal translation movement $(\mathrm{Hu}$ and $\mathrm{Wu}, 2002)$ and orthogonal movement method based on homographic matrix (Wu and $\mathrm{Hu}, 2001,2002$ ). 
The self-calibration of camera is a process that calibrating the camera by the corresponding relation of the image points, without any calibration blocks. In the process of self-calibration, it is assumed that the corresponding relation of the image points is determined, and the interior parameters of the camera make no changes when different images are shot (Yu et al., 1999; Wu et al., 2001).

\section{RESULTS FOR CAMERA CALIBRATION}

Since the machine vision system applied in nondestructive measurement of plants does not require the online calibration, the traditional linear calibration using calibration blocks can easily meet the testing requirements.

Therefore, calibration is done using a one-Yuan coin as a calibration reference. The calibration contains the processes below: collecting images of the calibration reference by CCD camera, shooting images in the condition that the vertical shooting height is fixed and the distance between the lens and the calibration reference and the distance between the lens and the plants are set at the same condition, counting the number of the pixels and calculating the true size that each pixel represents. According to the true size that each pixel represents, the true size of the plant shot in the same condition can be calculated. Figure 1 stands for the three images of two coins collected in different locations when the distance between the lens and the coin is $20 \mathrm{~cm}$ and the focal length is $16 \mathrm{~cm}$. Table 1 stands for the corresponding testing results.
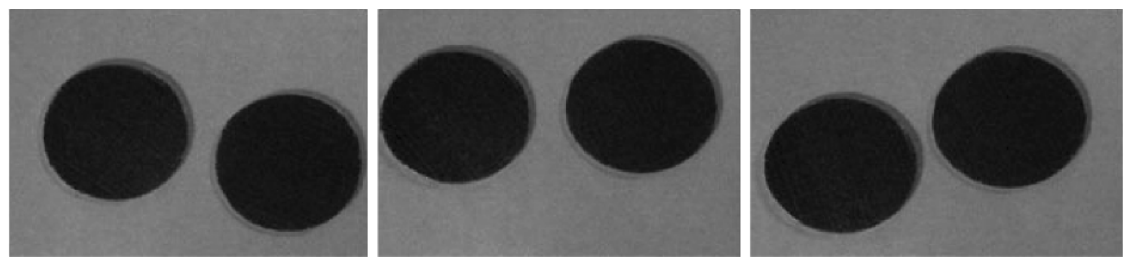

Figure 1. Image of coins

Table 1. Result of measurement

\begin{tabular}{lccc}
\hline Figure 1 & pixels & size per pixel $\left(\mathrm{mm}^{2}\right)$ & length per pixel $(\mathrm{mm})$ \\
\hline Left & 61231 & 0.00566 & 0.07523 \\
& 59802 & 0.00579 & 0.07609 \\
Middle & 61064 & 0.00567 & 0.07530 \\
& 59756 & 0.00579 & 0.07609 \\
Right & 61217 & 0.00566 & 0.07523 \\
& 60105 & 0.00576 & 0.07589 \\
Average & 60529 & 0.00572 & 0.07564 \\
\hline
\end{tabular}


As plants grow, calibration outcomes in different altitudes can be obtained when adjusting the distance between the lens and the coin, and through this, the values of plants can be calculated in the corresponding distance. The average size per pixel is $0.00572 \mathrm{~mm}^{2}$.

\section{REFERENCES}

$\mathrm{Hu} \mathrm{Z}$, Wu F. A review on some active vision based camera calibration techniques. Chinese Journal of Computer, 2002, 25 (11): 1149-1156.

Jain R, Kasturi R, Schunck. Machine vision. China Machine Press, Beijing: 1-20, 2003.

Tsai R Y. A versatile camera calibration technique for high-accuracy 3D machine vision metrology using off-the-shelf TV cameras and lenses. IEEE Journal of Robotics and Automation, 1987, 3 (4): 323-343.

Wu F, Hu Z. Linear determination of the infinite homography and camera self-calibration. Chinese Journal of Automation, 2002, 28 (4): 488-496.

$\mathrm{Wu} \mathrm{F}, \mathrm{Hu} \mathrm{Z}$. A new theory and algorithm of linear camera self calibration. Chinese Journal of Computer, 2001, 24 (11): 1121-1135.

$\mathrm{Wu} \mathrm{F}$, Li H, Hu Z. New active vision based camera self-calibration technique. Chinese Journal of Automation, 2001, 27 (6): 736-746.

$\mathrm{Wu} \mathrm{W}$, Sun Z. Overview of camera calibration methods for machine vision. Chinese Journal of Computer Applied Research, 2004, (2): 4-6.

Yang N, Yang J, Huang C, et al. Influence of calibration on the accuracy of 3-D reconstruction in direct linear transformation algorithm. Journal of Tsinghua University (Natural Science), 2000, 40 (4): 24-27.

$\mathrm{Yu} \mathrm{H}$, Wu F, Yuan B, et al. Camera self calibration technique based on active vision. Chinese Journal of ROBOT, 1999, 21 (1): 1-7.

Zhang Z. Computer vision -Theory and algorithms for calculating. Scientific publishing company, Beijing, 1998. 